\title{
Bulk micromachining technology for fabrication of two-level MEMS in standard silicon substrate
}

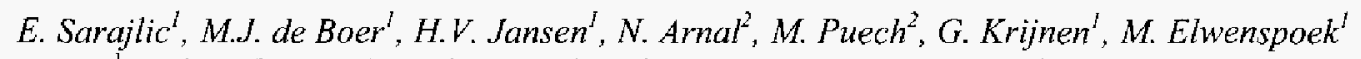 \\ ${ }^{1}$ MESA + Research Institute, University Of Twente, Enschede, The Netherlands \\ ${ }^{2}$ Adixen Micro Machining Systems, Alcatel Vacuum Technology, Annecy Cedex, France \\ e.sarajlic@el.utwente.nl
}

\begin{abstract}
We present a bulk micromachining technology, based on vertical trench isolation, for fabrication of two-level MEMS in a standard monocrystalline silicon substrate. The technology, suitable for full integration with on-chip electronics, employs double-side processing to obtain functional micromechanical structures on both sides of the substrate. Etching and release of these microstructures are performed in a single plasma chamber allowing dry, low temperature processing. Utilizing double-side processing, high-aspect-ratio monocrystalline MEMS with integrated vertical electrical insulation on fixed and even on movable parts are fabricated on one side of the wafer and, at the same time, mechanically and electrically coupled with released micromechanical structures on the opposite side. This opens new design opportunities to improve performance and functionality of MEMS devices. The presented technology is employed for fabrication of electrostatic XY-scanners for probe-based data storage with a significantly larger effective recording area proving the feasibility and large potential of this technology.
\end{abstract}

\section{INTRODUCTION}

Electrostatic $X Y$-scanners are considered well suited for positioning of a recording medium in a probe-based data storage system [1-2]. In general, these scanners consist of a suspended movable stage, driven by two pairs of combdrive microactuators in two orthogonal directions.

The clectrostatic $\mathrm{XY}$-scanners, reported until now, have been built in a single device layer, with all components at the same level, resulting in a relatively simple design. A serious drawback of the single-level electrostatic XYscanners is low area efficiency. The comb-drive actuators and spring suspension consume a large part of the total scanner area, leaving only a small part for the movable stage where the recording medium can be deposited. Therefore, only a relatively small portion of the total scanner area can actually be used for data storage. The area efficiency for the single-level electrostatic XY-scanners is typically less than $10 \%[2]$.

In this paper we present a bulk micromachining technology, based on standard single crystal silicon wafers and vertical trench isolation, that allows fabrication of electrostatic XY-scanners for probe-based data storage with a significantly larger effective recording area of $76 \%$.

\section{TECHNOLOGY}

The basic fabrication steps of the bulk micromachining technology are illustrated in Fig. 1.

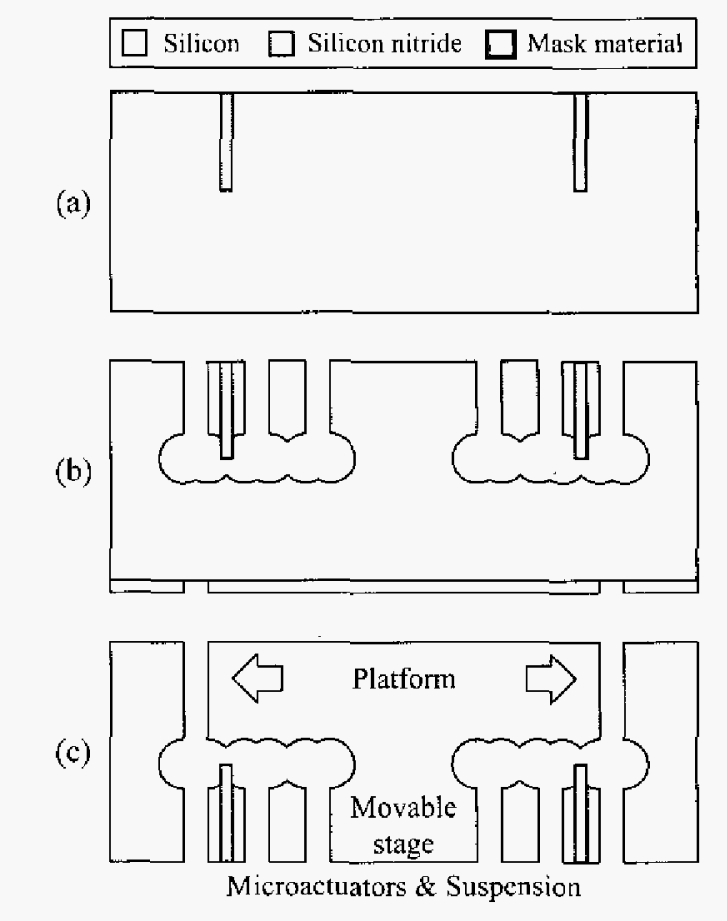

Figure 1: Condensed fabrication sequence: (a) trench etching and refill, (b) structural etching and plasma release and (c) backside etching.

A three-mask fabrication process starts on a standard single crystal silicon substrate with an arbitrary crystallographic orientation. In the silicon substrate, isolating trenches are etched by DRIE and subsequently refilled with a low stress LPCVD silicon nitride. The backfill material from the top surface of the substrate is then removed by a maskless directional etching step. The surface of the substrate remains flat and smooth after the etching, allowing further processing. This may include integration of on-chip electronics and/or embedded interconnects.

After removal of the backfill material from the top surface, the structural layout on the front side of the substrate is etched into the bulk silicon and subsequently released using an isotropic plasma etch to laterally undercut microstructures. We employ the advanced plasma processing [3] to etch, passivate and release microstructure in the same plasma system during the same etch run. This method allows the fabrication of released, high-aspect-ratio microstructures (sce Fig. 2) using dry, low temperature processing. 


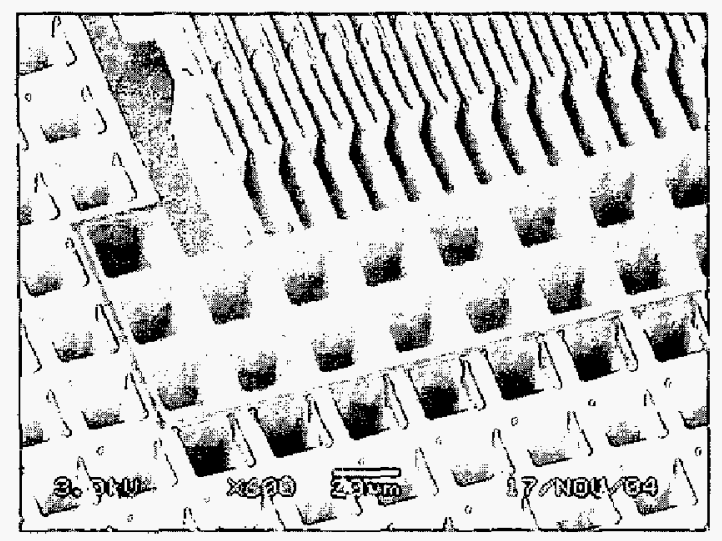

Figure 2: SEM micrograph of a released comb-drive with integratged vertical trench isolation

After the plasma release, deep reactive ion etching is performed on the flipped wafer all the way through the remaining silicon layer located beneath the microstructures previously machined on the front side of the substrate. The microstructures on the front side are protccted with a fluorocarbon (FC) that is uniformly deposited in the same plasma system prior the backside etching.

\section{TWO-LEVEL XY-SCANNER}

The bulk micromachining technology, presented in the previous section, utilizes both sides of the substrate allowing fabrication of a two-level XY-scanner (Fig. Ic). A movable stage of this scanner, suspended by a spring suspension and driven by microactuators, is mechanically connected to a platform machined on the opposite side of the substrate. The non-perforated platform, on which a recording medium is to be applied, completely covers the area used for the microactuators and the spring suspension.

By hiding the driving components beneath a movable platform covered by the recording medium, a large area required for these components can be efficicntly used for the data storage. This also allows further miniaturization, and thereby an increase in speed, of the medium scanners without compromise of the recording area efficiency. Furthermore, the microactuators in a two-level XY-scanner are placed on the opposite side of the recording medium, preventing a potentially harmful influence of the microactuators on the operation of micromechanical probes used for the recording and vice versa.

\section{FABRICATION AND RESULTS}

We demonstrate the feasibility of the proposed technology by fabricating and operating a two-level $X Y$-scanner, shown in Fig. 3. Etching and release steps are performed in a state of the art etching machine type AMS 100SE of the company ADIXEN [4].

The scanner fits in an area of $2412 \mu \mathrm{m} \times 2412 \mu \mathrm{m}$. A movable stage is actuated electrostatically by two pairs of comb-drive microactuators. Four intermediatc stages and a folded flexure suspension are employed to provide a decoupled motion of the central stage along two orthogonal axes. The movable stage and the intermediate stages are suspended by folded flexures.

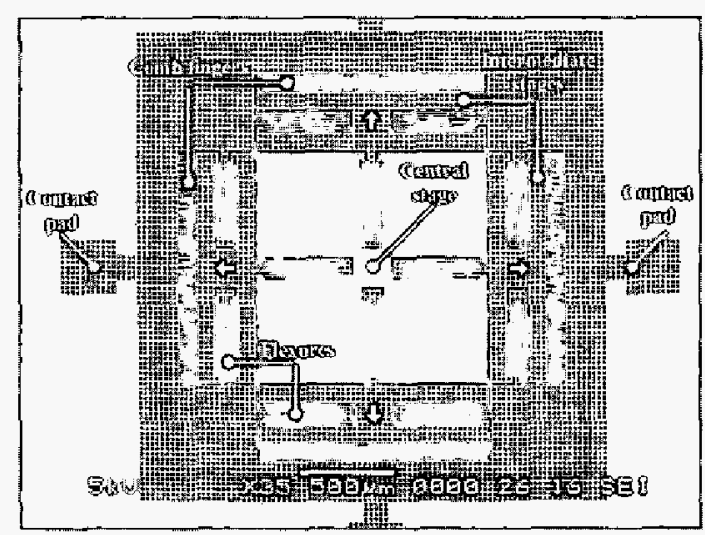

Figure 3: SEM micrograph of a two-level XY-scanner.

The central stage is mechanically coupled to a $2106 \mu \mathrm{m} \mathrm{x}$ $2106 \mu \mathrm{m}$ platform machined on the opposite side of the substrate. The non-perforated rectangular platform, shown in Fig. 4, occupies $76 \%$ of the total scanner area.

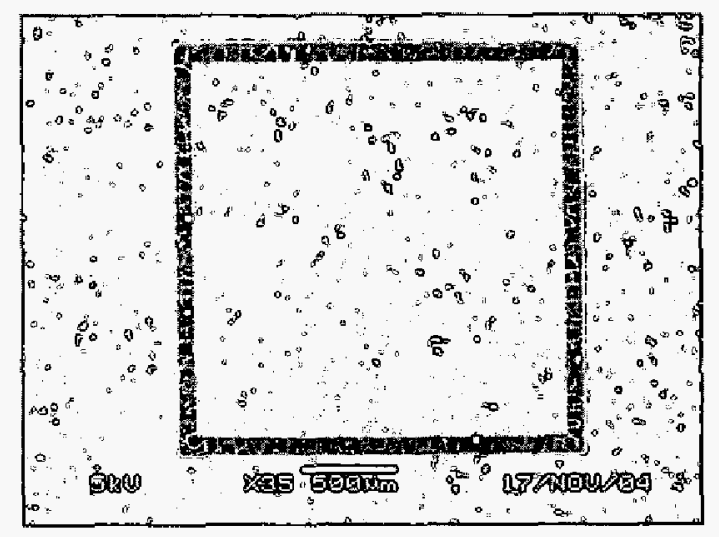

Figure 4: SEM micrograph of a released platform. All driving components of $X Y$-scanner are hiden beneath.

The two-level XY-scanner was successfully operated, demonstrating a decoupled motion along two axes.

\section{CONCLUSION}

A bulk micromachining method for fabrication of doublesided MEMS on standard monocrystalline silicon substrate is presented. The technology is successfully employed for fabrication of an electrostatic XY-scanner for probe-based data storage with a significantly large area efficiency of 76 $\%$, proving the feasibility and large potential.

\section{REFERENCES}

[1] Carley L R et al $2000 J$ of Applied Physics 87 6680-5

[2] $\mathrm{Kim} \mathrm{C} \mathrm{H}$ et al $2003 \mathrm{~J}$. Microelectommech. Syst. 12(4) $470-8$

[3] Sarajlic E et al 2004 J.Micromech. Microeng. 14 S70-5

[4] Adixen Micro Machining Systems ( $w$ ww:adixen.com) 